\title{
PROSTHORHYNCHUS CYLINDRACEUS (Goeze 1782) AU STADE JUVÉNILE, CHEZ UN ISOPODE TERRESTRE ACANTHOCEPHALA-POLYMORPHIDAE
}

\author{
Par Robert Ph. Dollfus et Henri DALENS
}

Chez des Armadillidium vulgare Latreille, dont la plupart provenaient d'un élevage [souche récoltée dans un jardin, à Toulouse (Haute-Garonne)], une infestation massive par Acanthocéphale a été constatée par l'un de nous (M. D.), en avril 1959. Il est, en général, possible de savoir, par l'examen des cloportes vivants, quels sont ceux parasités ; les parasites forment, en effet, dans l'abdomen de l'hôte, des taches blanches, visibles par transparence. Dans un lot de 11 cloportes (tant $\delta^{\star}$ que q) parasités, dont 10 prélevés dans l'élevage, il y en avait neuf qui, d'après l'examen extérieur, ne montraient qu'une seule tache blanche, ce qui indiquait la présence d'au moins un parasite. Le $10^{\circ}$ cloporte contenait deux parasites, situés à la base de la cavité du péréion, de part et d'autre du tube digestif. Le $11^{\circ}$ hébergeait quatre parasites, de tailles inégales, situés du même côté du tube digestif, remplissant toute la partie droite de la cavité péréiale. Aucun n'était enkysté.

Le parasite se présente comme un corps ellipsoïde-piriforme d'environ 1,5 à $3 \mathrm{~mm}$. de long et 0,25 à 0,35 de large, dont l'extrémité antérieure se prolonge par un proboscis cylindrique, long en moyenne d'environ $1 \mathrm{~mm}$., avec un diamètre de 0,19-0,22 $\mathrm{mm}$. Chez un individu, les crochets sont disposés en 13 files longitudinales de 12-13, dont les deux derniers, à racine très rudimentaire, sont, en fait, des épines. Chez un autre, il y a 16 files de 14 . Dans chaque file, la longueur des crochets, depuis l'apex, augmente jusqu'à environ le $7^{\bullet}(0,07 \mathrm{~mm}$.$) , puis diminue, et les épines sont longues seu-$ lement de 55-60 u.

Le ganglion nerveux est situé dans le tiers moyen du réceptacle du proboscis et le réceptacle s'insère au niveau de sa jonction avec le cou, c'est-à-dire juste en arrière de la dernière rangée circulaire d'épines. Les spécimens $\hat{\sigma}$ sont pourvus de testicules et de glandes cémentaires, non encore fonctionnels. Les spécimens $q$ contien- 


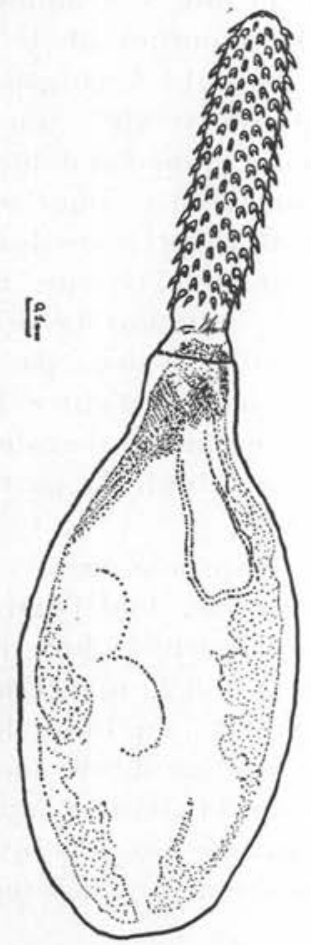

Fig. 1. - Individu in toto.

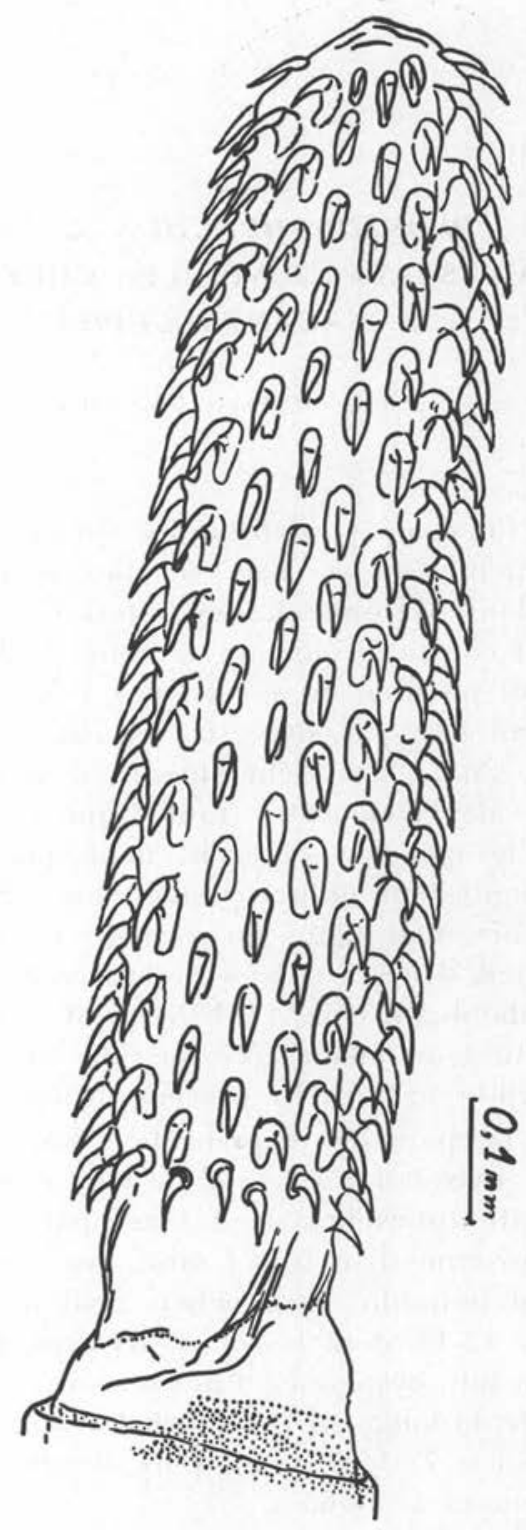

FIG. 2. - Proboscis évaginé.

nent des masses ovariennes en voie de développement, mais pas d'œufs libres avec coque. Il s'agit de la larve âgée, dite «stade juvénile », d'un Acanthocéphale dont l'anatomie et la morphologie, externe sont celles des Prosthorhynchus Kostylev 1915. 
Environ 17 espèces ont été placées dans ce genre, mais, en raison des synonymies, il n'y a que neuf espèces valables, d'après la révision du genre par Y.-J. Golvan (1956, p. 357-367). Nous rapportons les spécimens trouvés chez Armadillidium à Prosthorhynchus cylindraceus (Goeze 1782), espèce à caractères quelque peu variables, qui a reçu au moins 13 noms spécifiques différents. Généralement, chez cylindraceus, le nombre des files longitudinales de crochets est de 14 à 18 (mais peut atteindre 24 ) et il y a 10 à 18 crochets dans chaque file, dont les deux ou trois inférieurs sont des épines. Bien qu'un de nos spécimens ait seulement 13 files longitudinales de 12-13 crochets (y compris les épines), nous estimons, étant donnée la variabilité de l'espèce, que nos spécimens correspondent à cylindraceus, seule espèce du genre connue en Europe Occidentale, où elle est adulte chez environ une trentaine d'espèces de Passériformes terrestres à régime alimentaire au moins en partie insectivore.

La larve (au stade dit juvénile) d'une espèce voisine, Prosthorhynchus formosus (Van Cleave 1918), a été identifiée dans un Armadillidium vulgare Latreille, des environs de Washington (D. C.), par D. Sinitsin (1929, p. 287) ; cette larve était longue de $4 \mathrm{~mm}$.

Il est donc vraisemblable que, dans le genre Prosthorhynchus, l'hôte intermédiaire est un Arthropode terrestre.

Chez Armadillidium vulgare Latr., Porcellio scaber Latr., Philoscia muscorum (Scopoli), des larves d'Acanthocéphales ont été signalées par W. R. Thompson (1934, p. 433, 440); elles n'ont malheureusement pas été identifiées et leur lieu de récolte (France ?, Grande-Bretagne ?) n'a pas été précisé.

Des larves d'Acanthocéphales ont été signalées chez des Isopodes aquatiques beaucoup plus souvent que chez des terrestres.

\section{OUVRages CITÉs}

Golvan (Yves J.), 1956. - Acanthocéphales d'Oiseaux. Troisième note. Révision des espèces européennes de la sous-famille des Plagiorhynchina A. Meyer 1931 (Polymorphidæ). Annales de Parasitol. hum. et compar.. t. XXXI, n 4,1956 , p. 350-384, fig. texte 1, pl. I-IV.

SinITSiN (Dimitri P.), 1929. - An intermediate host for Plagiorhynchus formosus Van Cleave. The Journ. of Parasitol., v. XV, $\mathrm{n}^{\circ} 4,27-8-1929$, p. 287.

Thompson (William Robin), 1934. - The Tachinid parasites of Woodlice. Parasitology, Cambridge, v. XXVI, $\mathrm{n}^{\circ} 3,17-8-1934$, p. 378-400, fig. texte 1-5, pl. XV-XXII, fig. 1-79, 